\title{
Les extractions de matériaux dans le lit mineur et le lit majeur de la Loire et de ses affluents
}

\author{
par Jean-Louis DAMBRE \\ Ingénieur général des Ponts et Chaussées honoraire
}

\section{I 圆 INTRODUCTION}

La Loire a la réputation d'être un fleuve naturel, voire "sauvage". En fait, au cours des siècles, elle a fait l'objet de nombreux aménagements (levées, sensiblement parallèles aux berges, pour la protection contre les inondations, et ouvrages pour faciliter la navigation), dont la plupart subsistent encore.

En outre, d'importantes extractions de matériaux (sables et graviers) ont été effectuées dans son lit mineur et son lit majeur, ainsi que dans ceux de ses affluents, notamment après la dernière guerre, pour les besoins de la reconstruction et du développement économique du pays.

Ces aménagements et ces extractions dans le lit mineur ont provoqué l'approfondissement du lit de la Loire et l'abaissement de la ligne d'eau en étiage. A titre d'exemple, celle-ci a baissé, depuis le début du siècle, d'environ $2 \mathrm{~m}$ à Tours, $3 \mathrm{~m}$ à Ancenis et $4 \mathrm{~m}$ à Bellevue, à l'amont de Nantes.

Les conséquences de cette situation sont particulièrement graves pour les ouvrages (déchaussement des piles de pont et des perrés, voire effondrement de ceux-ci), les abords du fleuve et l'environnement (dessèchement des bras secondaires, des boires et des zones humides, abaissement du niveau de la nappe phréatique, diminution des frayères, perturbation de la faune et de la flore...).

L'aggravation de cette situation, à la fin des années soixante-dix, a conduit le ministre de l'Environnement à engager dès 1980, suite au rapport FOUCAUD, une politique de réduction progressive des extractions de matériaux dans le lit mineur de la Loire et de l'Allier, puis à fixer en décembre 1984 comme objectif l'arrêt complet de ces extractions fin 1992.

A la demande du ministre de l'Environnement, une enquête a été lancée en 1992 pour évaluer les conditions de poursuite de la politique de limitation des extractions de matériaux dans le lit de la Loire, de Bec d'Allier à Nantes.

Cette enquête a été confiée à Pierre Malaval, IGGREF, et à moi-même, tous deux membres de la mission d'inspection spécialisée de l'Environnement (MISE), et a donné lieu au rapport $\mathrm{n}^{\circ}$ 92-221 du 20 juillet 1993.

Suite à ce rapport, le directeur de l'eau a demandé d'étendre cette enquête sur les extractions de matériaux à l'ensemble du lit mineur et du lit majeur de la Loire et de ses affluents.

Cette enquête m'a été confiée et a donné lieu au rapport n $93-232$ du 2 mai 1995.

La présente communication consiste dans la présentation succincte de ces deux rapports et des décisions prises pour une approche globale des problèmes ligériens, mettant fin aux désordres constatés.

\section{1 LES EXTRACTIONS DE MATERIAUX EN LOIRE ENTRE BEC D'ALLIER ET NANTES}

\subsection{Le constat}

De la première enquête, il ressort les constatations suivantes :

- les extractions de matériaux dans le lit mineur ont été considérables puisqu'elles se sont élevées, entre 1949 et 1992, à 220 millions de tonnes, soit sensiblement l'équivalent d'une bande de $150 \mathrm{~m}$ de largeur et de $1,50 \mathrm{~m}$ de hauteur en Loire moyenne et d'une bande de $200 \mathrm{~m}$ de largeur et de 2,40 m de hauteur en Basse-Loire ;

- les extractions dans le lit mineur ont été croissantes de 1960 à 1979, passant d'environ 2 à 13 millions de tonnes/an ;

- ces extractions ont chuté dès 1980, suite à une lettre-circulaire du 12 juin 1980 des ministres de l'Environnement et du Cadre de Vie, et des Transports aux préfets, leur demandant de réduire progressivement de façon très sensible les extractions de matériaux dans le lit des cours d'eau du domaine public fluvial, et au protocole du 8 mai 1981 relatif aux extractions de matériaux dans le lit mineur de la Loire et de l'Allier, passé entre les deux ministres précités, le ministre de l'Industrie et la profession des exploitants sabliers pour réduire les extractions de matériaux dans le lit mineur de la Loire et de l'Allier.

A partir de 1986, ces extractions se sont stabilisées autour de 3,8 millions de tonnes/an jusqu'en 1991. Elles ont cessé dans le lit mineur de la Loire et de l'Allier, début 1993, sauf dans les trois départements de l'aval (I'Indre et Loire, le Maine et Loire et la Loire Atlantique), où elles étaient les plus importantes. De plus, ces trois départements étant pauvres en matériaux de substitution, les problèmes de 
reconversion des entreprises vers d'autres gisements y étaient plus difficiles à résoudre que dans le reste du bassin. - les extractions dans le lit majeur de la Loire moyenne ont fortement augmenté de 1981 à 1991, passant de 857000 tonnes à 3116000 tonnes. Cette augmentation correspond à $82 \%$ de la diminution des extractions dans le lit mineur de cette section.

\subsection{Les conséquences des extractions de matériaux dans le lit mineur}

\subsubsection{Les extractions dans le lit mineur actif}

Les fosses réalisées en Loire pour les extractions de matériaux provoquent un abaissement de la ligne d'eau amont, donc une érosion régressive, souvent considérée comme la principale conséquence d'un prélèvement.

Cependant, le débit solide provenant de l'amont se bloque au moins partiellement dans la zone draguée. A l'aval, le cours d'eau reconstitue le débit solide, correspondant à sa capacité de transport, aux dépens des matériaux de son lit, provoquant un abaissement du fond avec diminution de pente par érosion progressive.

S'il s'agit d'une extraction temporaire, le débit solide amont finit par combler la fosse. Mais les conditions amont n'ayant pas changé, la compensation des matériaux enlevés se fait par prélèvement dans le lit et se traduit par un approfondissement réparti.

Par ailleurs, les crues importantes mobilisent une forte épaisseur de sédiments et tendent à niveler les fonds en répartissant l'approfondissement dans chaque bief. Cet approfondissement est atténué par les matériaux provenant de l'érosion des berges et des îles I.

Dans tous les cas, une extraction, aussi faible soit-elle, se traduit par un approfondissement réparti. L'argument de l'innocuité de prélèvements inférieurs à la valeur du débit solide, avancé parfois, n'est pas recevable, cette valeur n'ayant d'effet que sur le délai de répartition de l'abaissement et non sur son importance moyenne.

\subsubsection{Les prélèvements dans les bras secondaires}

Dans les secteurs où le lit comporte plusieurs bras, il est fréquent qu'un ou plusieurs bras secondaires soient ensablés et envahis par la végétation, soit naturellement, soit par suite de l'existence d'ouvrages de concentration du débit en vue de faciliter la navigation.

La capacité de transport est plus importante dans le lit principal, ce qui contribue à une différenciation croissante du niveau des fonds. La diminution des vitesses et le développement de la végétation accentuent ce phénomène, en favorisant les dépôts de limons.

\footnotetext{
1 La proportion des matériaux provenant du fond serait de l'ordre de $70 \%$ sur une longue période dans la zone de Tours d'après une étude d'HYDRATEC de septembre 1985 : Etude hydraulique et de morphologie fluviale.
}

Une idée courante est qu'il s'agit de "bras morts", qui ne participent plus à la dynamique du fleuve. Il n'y aurait donc aucun inconvénient à y pratiquer des extractions importantes, en barrant chaque extrémité par des seuils pour éviter les interactions avec le fleuve lui-même.

En fait, la capacité de transport de ces bras est loin d'être négligeable. Bien qu'elle soit plus faible que celle du chenal principal, la hauteur d'eau étant moins importante, il se produit, lors des crues, une érosion, qui est favorisée par la présence d'un seuil à l'amont. La partie du débit solide qui est piégée, contribue en effet au déficit de celui-ci, donc à l'érosion en aval. Les extractions dans les bras secondaires contribuent à l'abaissement du fond du lit principal de la même façon que celles effectuées dans celui-ci.

Enfin, il convient de ne pas oublier que l'inversion de la tendance à l'approfondissement nécessite des apports de matériaux, qui doivent provenir évidemment d'autres sources que l'approfondissement du lit principal. Ils peuvent provenir des berges, qu'il faut se garder de protéger quand des intérêts socio-économiques majeurs ne sont pas en jeu, et des dépôts dans les bras secondaires, qu'il ne faut donc pas prélever à d'autres fins.

Il convient donc de ne pas extraire, même avec précautions, les matériaux localement excédentaires, mais de les fournir à l'aval en procédant le plus possible à un transfert dans le lit principal, soit artificiellement, soit en favorisant l'érosion naturelle.

\subsubsection{L'enlèvement des atterrissements}

Des atterrissements importants peuvent diminuer la capacité d'écoulement des crues ou donner au chenal principal une orientation entraînant l'érosion d'une berge, que l'on désire protéger.

L'enlèvement d'un atterrissement permet un "rééquilibrage hydraulique". Mais, si les conditions amont et aval ne sont pas modifiées, il se reformera lors d'une crue, en provoquant une érosion aval compensant l'immobilisation de son volume. Pour que la modification d'un atterrissement ou son enlèvement n'ait pas de conséquence sur l'approfondissement du lit, il convient d'opérer un transfert de matériaux dans le lit principal.

\subsubsection{Les conséquences de l'aménagement de l'estuaire}

L'approfondissement du chenal de navigation maritime depuis le début du siècle et les extractions importantes réalisées à Nantes pour fournir des matériaux ont considérablement diminué "l'inertie hydraulique" de l'estuaire. Le niveau des basses mers à l'amont immédiat de Nantes a diminué d'environ 4 mètres depuis 1900.

D'autre part, les extractions à l'amont ont contribué à étendre l'influence des hautes mers sur une trentaine de kilomètres du fleuve.

A marée haute, la capacité de transport est probablement diminuée, l'ěcoulement naturel étant davantage freiné. Mais, au jusant, cette capacité a considérablement augmenté depuis le début du siècle. La pente hydraulique disponible 
depuis Ancenis a presque doublé et l'évacuation d'une quantité d'eau très importante, en raison de l'extension du marnage vers l'amont, s'ajoute au débit naturel.

Le débit atteint ainsi fréquemment des valeurs de $2000 \mathrm{~m}^{3} / \mathrm{s}$ environ, du même ordre de grandeur que celui de la crue annuelle. Non seulement le débit solide a fortement augmenté, mais il "fonctionne" beaucoup plus fréquemment Il s'ensuit une érosion régressive intense, que l'émergence à Mauves d'un seuil rocheux ne suffit pas à interrompre.

\subsubsection{Un fleuve déséquilibré}

On admet que les apports externes de sables et graviers sont devenus très inférieurs à la capacité de transport du fleuve (évolution de l'agriculture, reboisement dans le Massif Central, barrages amont). La tendance à l'érosion des berges et du fond à partir de l'amont serait permanente, la pente d'équilibre étant loin d'être atteinte.

Les mesures anciennes montrent une tendance lente à l'abaissement du lit, accélérée depuis une trentaine d'années par les extractions massives de matériaux et, plus localement, par l'abaissement du niveau des basses mers à l'entrée de l'estuaire.

D'autre part, l'encaissement du lit mineur réduit fortement la fréquence de débordement, augmentant la capacité de transport des crues importantes et "améliore" l'efficacité des ouvrages de concentration du débit. L'approfondissement a un effet cumulatif.

\subsection{Les propositions du rapport du 20 juillet 1993}

Compte tenu des conséquences dommageables pour l'environnement de l'abaissement de la ligne d'eau en étiage, le rapport propose comme objectif principal d'inverser la tendance actuelle et de mettre en oeuvre une politique pour faire remonter la ligne d'eau en étiage, sans artificialiser le fleuve, ce qui interdit une régulation de la ligne d'eau en étiage par des ouvrages, qui créeraient une succession de biefs

En ce qui concerne les extractions, les propositions du rapport sont les suivantes :

\section{- Arrêt des extractions dans le lit mineur}

L'étude de la dynamique du lit de la Loire montre que toute extraction de matériaux dans le lit mineur, même dans les bras morts, soit contribue directement à l'abaissement du lit, soit prive le fleuve des alluvions nécessaires à un relèvement ultérieur

Il est donc indispensable :

- de mettre fin aux extractions de matériaux dans le lit mineur au titre du code minier, l'obtention de matériaux de substitution s'avérant possible, bien qu'entraînant une augmentation des coûts. Les protocoles entre l'Etat et les représentants des exploitants sabliers, négociés en 1992, ont prévu cet arrêt : fin août 1993 pour la Loire Atlantique, fin 1993 pour le Maine et Loire et fin 1995 pour l'Indre et Loire,

- de restituer au fleuve les matériaux extraits à d'autres fins (dégagement de prises d'eau, réactivation de bras secondaires, déplacement du courant principal, amélioration de l'écoulement des crues...). Il convient soit d'opérer des transferts, soit de favoriser l'érosion naturelle. Des instructions fermes devraient être données à ce sujet, les évolutions locales du lit risquant de multiplier les nécessités d'intervention.

\section{- Réduction des extractions dans le lit majeur}

Le remplacement des extractions dans le lit mineur par des extractions dans le lit majeur ne peut être que partiel et transitoire. Si l'on peut envisager en Basse-Loire de compléter les approvisionnements en sable marin par l'ouverture de quelques carrières dans le lit majeur, il convient d'être très prudent en Loire moyenne. La diminution des prélèvements dans le lit mineur y a été, en moyenne, compensée par des extractions dans le lit majeur à hauteur de plus de $80 \%$. La surface des carrières qui seront transformées à terme en plans d'eau est déjà considérable et ne saurait beaucoup augmenter sans transformation profonde des équilibres écologiques et des paysages. Il est nécessaire d'affirmer une volonté de freinage, d'intensifier les recherches techniques pour l'emploi de matériaux de substitution et de prospecter les gisements correspondants.

En outre, le rapport fait des propositions concernant l'entretien, la restauration et la gestion du lit de la Loire, ainsi que la compatibilité de l'objectif de remontée de la ligne d'eau en étiage avec le maintien des ouvrages de navigation.

\subsection{Les décisions prises à la suite de ce rapport}

Le Plan-Loire, décidé par le Gouvernement le 4 janvier 1994, vise à concilier la sécurité des personnes, la protection de l'environnement et le développement économique. Ce plan comporte un certain nombre de mesures proposées dans le rapport, notamment en ce qui concerne le relèvement de la ligne d'eau en étiage

"Afin d'assurer la sécurité de l'alimentation en eau des collectivités et restaurer l'équilibre du fleuve, le Gouvernement considère comme prioritaire l'objectif de relèvement de la ligne d'eau en étiage de la Basse-Loire et de la Loire moyenne.

Le Gouvernement rappelle qu'il a décidé l'arrêt définitif de l'extraction des granulats dans le lit mineur de la Loire. Dans le lit majeur, des limitations seront arrêtées dans le cadre du schéma directeur d'aménagement et de gestion des eaux et des schémas départementaux des carrières".

Des instructions ont été adressées aux préfets pour la mise en oeuvre de cette décision.

En outre, les dommages causés à l'environnement par les extractions de matériaux n'étant pas propres qu'à la Loire, le ministre de l'Environnement a interdit par arrêté du 22 septembre 1994, relatif aux exploitations de carrières, les extractions dans le lit mineur des cours d'eau et des plans d'eau traversés par des cours d'eau. Les seules extractions qui peuvent être autorisées, sont celles nécessaires à l'entretien du lit dûment justifié ou à l'aménagement d'un cours d'eau.

En ce qui concerne les cours d'eau à régime torrentiel, ayant un débit solide important, les modalités d'application 


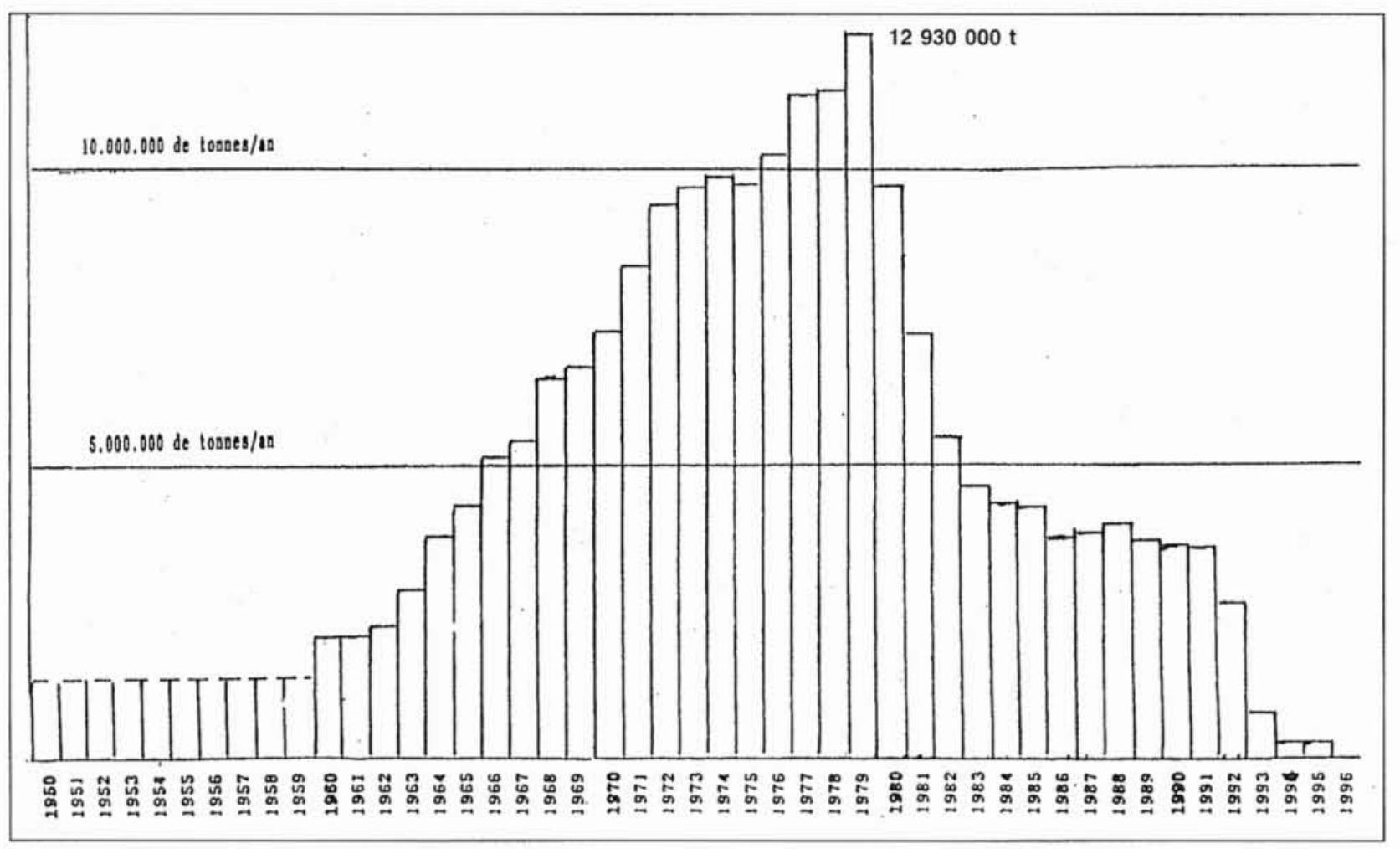

1. Extraction de matériaux dans le lit mineur de la Loire entre Bec d'Allier et Nantes.

\begin{tabular}{|c|c|c|c|c|c|c|c|c|c|c|c|c|c|}
\hline \multirow{3}{*}{ Ammées } & \multicolumn{5}{|c|}{$\begin{array}{c}\text { BASSIN DE LA LOIRE } \\
\text { A L'AMONT DE BEC D'ALLIER }\end{array}$} & \multicolumn{7}{|c|}{$\begin{array}{c}\text { BASSIN DE LA LOIRE } \\
\text { A L'AVAL DE BEC D'ALLIER }\end{array}$} & \multirow{3}{*}{$\begin{array}{l}\text { TOTAL } \\
\text { GENERAL }\end{array}$} \\
\hline & \multirow{2}{*}{$\begin{array}{c}\text { La } \\
\text { Loire }\end{array}$} & \multicolumn{3}{|c|}{ Affluents de la Loire } & \multirow{2}{*}{$\begin{array}{l}\text { TOTAI. } \\
\text { (I) }\end{array}$} & \multirow{2}{*}{$\begin{array}{c}\text { La } \\
\text { Loire }\end{array}$} & \multicolumn{5}{|c|}{ Affluents te la Loire } & \multirow{2}{*}{$\begin{array}{c}\text { TOTAL. } \\
\text { (II) }\end{array}$} & \\
\hline & & $\begin{array}{l}\text { Rassin ale } \\
\text { IAlller }\end{array}$ & Arrourx & sthotul & & & C'her & Intre & $\begin{array}{l}\text { Massin de } \\
\text { la I'iemue }\end{array}$ & $\begin{array}{l}\text { Alussin de } \\
\text { la Maine }\end{array}$ & s/total & & \\
\hline 1981 & 3116 & 4232 & 366 & 4598 & 7711 & 987 & 197 & 160 & 45 & 0 & 1402 & 2,380 & 16 103 \\
\hline 1982 & 3093 & 3635 & 393 & | & 7121 & 968 & 1327 & 218 & 132 & 0 & 1677 & 2645 & 9) 766 \\
\hline 1983 & 2687 & 3502 & 416 & 3948 & 66.15 & 2816 & 1261 & 152 & 115 & 0 & 1528 & 4344 & 10979 \\
\hline 1984 & 2132 & 2825 & 207 & 3122 & 5254 & 2123 & 1362 & 171 & 126 & 20 & 1679 & 3802 & 9056 \\
\hline 1985 & 1984 & 2803 & 3Rs & 3191 & 5175 & 1945 & 1211 & 154 & 178 & 407 & 1950 & 3 8\%5 & 9676 \\
\hline 1986 & 2091 & 3588 & 358 & $3 \% 66$ & 60.37 & 2698 & 1355 & 156 & 134 & 490 & 2135 & 4813 & 16870 \\
\hline 1987 & 2201 & 3605 & 317 & 39.12 & 6.1 .17 & $308-1$ & 1989 & 123 & 160 & 680 & 2952 & 6036 & 12183 \\
\hline 1988 & 2.407 & 3254 & 353 & 3607 & 6014 & 3327 & 2970 & 182 & 183 & $65 \mathrm{~B}$ & 3903 & 7.120 & 13334 \\
\hline 19Rי) & $277 \mathrm{x}$ & 34.32 & 36.3 & 3795 & 6.573 & 3435 & 1653 & 154 & 189 & 1268 & 3264 & 6699 & 13272 \\
\hline בים & 2119 & $3(90)$ & 361 & 1060 & 61790 & 325.4 & 316 & 9פי & 154 & 1220 & 2389 & 5643 & 11822 \\
\hline 1991 & 2217 & 3075 & 332 & 3.107 & 562.4 & 3201 & 1003 & 60 & 2001 & 1261 & 2528 & 5733 & II 357 \\
\hline 1992 & 2090 & 1238 & 387 & 3625 & 5715 & 3217 & 986 & 73 & 325 & 2410 & 3794 & 7011 & 12726 \\
\hline 1993 & 2088 & 2912 & 341 & 325,3 & 5.341 & 4113 & 924 & 99 & 350 & 21.13 & 3516 & 7629 & 12970 \\
\hline \begin{tabular}{|l|} 
rotus. \\
\end{tabular} & 31007 & $438(x)$ & 4722 & $4 \times 522$ & 79 529 & 35172 & 18154 & 1801 & 2295 & 10557 & $32 \mathrm{8m}$ & | פירע 67 & 147508 \\
\hline
\end{tabular}

2. Extractions de matériaux dans le lit majeur de la Loire et de ses affluents.

Tableau récapitulatif général (présentation par sous-bassin — en milliers de tonnes). 


\begin{tabular}{|l|r|r|r|}
\hline Années & $\begin{array}{c}\text { Lit mineur de } \\
\text { la Loire et de } \\
\text { ses affuents }\end{array}$ & $\begin{array}{c}\text { Lit majeur de } \\
\text { la Loire et de } \\
\text { ses affuents }\end{array}$ & TOTAL \\
\hline 1981 & 9687 & 10103 & 19790 \\
\hline 1982 & 6789 & 9766 & 16555 \\
\hline 1983 & 5866 & 10979 & 16845 \\
\hline 1984 & 5236 & 9056 & 14292 \\
\hline 1985 & 5060 & 9070 & 14130 \\
\hline 1986 & 4393 & 10870 & 15263 \\
\hline 1987 & 4598 & 12183 & 16781 \\
\hline 1988 & 4375 & 13334 & 17709 \\
\hline 1989 & 4139 & 13272 & 17411 \\
\hline 1990 & 3837 & 11822 & 15659 \\
\hline 1991 & 3780 & 11357 & 15137 \\
\hline 1992 & 2787 & 12726 & 15513 \\
\hline 1993 & 912 & 12970 & 13882 \\
\hline \hline$T O T A L$ & 61459 & 147508 & 208967 \\
\hline
\end{tabular}

3. Evolution des extractions de matériaux alluvionnaires dans le lit mineur et le lit majeur de la Loire et de ses affluents. (En milliers de tonnes).

sont précisées par l'article 29 de la loi $n^{\circ}$ 95-101 du 2 février 1995 sur le renforcement de la protection de l'environnement.

\section{III [ LES EXTRACTIONS DE MATERIAUX DANS L'ENSEMBLE DU BASSIN DE LA LOIRE}

\subsection{Le constat}

De la deuxième enquête, il ressort les constatations suivantes :

- les extractions de matériaux dans le lit mineur de la Loire et de ses affluents ont maintenant cessé, sauf dans le département d'Indre et Loire, où elles devraient être arrêtées prochainement. Il ne reste actuellement qu'un seul exploitant dans le lit mineur de la Vienne dans ce département.

- les extractions de matériaux dans le lit majeur de la Loire et de ses affluents ont augmenté de près de $30 \%$ en 12 ans, passant globalement de 10103000 t en 1981 à près de $13000000 \mathrm{t}$ en 1993.

L'analyse de cette évolution par sous-bassin est très contrastée. En effet :
- dans le sous-bassin de la Loire à l'amont de Bec d'Allier, les extractions dans le lit majeur ont diminué d'environ $30 \%$ de 1981 à 1993, passant de $7714000 \mathrm{t}$ à $5341000 \mathrm{t}$.

- mais, dans le sous-bassin de la Loire à l'aval de Bec d'Allier, elles ont très fortement augmenté $(+220 \%)$, au cours de cette période, passant de $2389000 \mathrm{t}$ à $7629000 \mathrm{t}$. Le report des extractions du lit mineur dans le lit majeur s'est effectué globalement dans ce sous-bassin à hauteur de $66,3 \%$.

La forte croissance des extractions dans le lit majeur de la Loire et de ses affluents à l'aval de Bec d'Allier doit être rapidement maîtrisée, car ces extractions ont un impact important sur l'environnement.

\subsection{Les propositions du rapport du 2 mai 1995}

Les propositions du rapport s'inscrivent tout à fait dans la ligne des recommandations du rapport de juin 1994 de l'instance d'évaluation des politiques publiques en matière de zones humides ', présidée par le préfet Paul Bernard, qui a mis en évidence la dégradation continue de la plupart des zones humides en France. La moitié d'entre elles ont disparu au cours des 30 dernières années.

Ces propositions sont les suivantes :

- Les extractions effectuées dans la partie du lit majeur située dans le lit endigué ou, lorsqu'il n'y a pas de digue, dans la partie du lit majeur correspondant à la crue décennale, ont un impact fort sur les zones humides. Pour la préservation de celles-ci, il est proposé de ne plus accorder dans ces zones de nouvelles autorisations d'extraction, ni de renouvellement, ni d'extension d'autorisation existante. L'arrêt des exploitations actuelles dans ces zones devrait être programmé à échéance de 10 ans, compte tenu des critères économiques d'amortissement des installations.

- En ce qui concerne la partie du lit majeur, en dehors du lit précédemment défini, une attention particulière doit être portée à la préservation des aquifères, des sites et des paysages. L'étude d'impact préalable à toute nouvelle autorisation devra montrer la compatibilité de l'ouverture d'une carrière avec l'environnement. Une réduction de $4 \%$ par an des extractions dans cette zone est proposée, conformément aux recommandations du rapport Barthelemy de juillet 1993 sur les schémas départementaux de carrières.

- Des protocoles devraient être établis dans chaque région par les préfets de région et les représentants des exploitants sabliers pour programmer l'arrêt des extractions dans le lit endigué ou le lit majeur délimité par la crue décennale et limiter les extractions dans le reste du lit majeur. Ces protocoles constitueraient des documents de référence pour l'établissement des schémas départementaux des carrières.

- Un suivi de la réduction de ces extractions devrait être effectué chaque année par la Mission Déléguée de Bassin.

— La gestion des matériaux alluvionnaires devrait être plus

1 Ce rapport souligne les dommages qui résultent pour le milieu naturel de la dégradation des zones humides et la nécessité de mettre en oeuvre une politique volontariste en faveur de ces zones pour arrêter leur dégradation. 


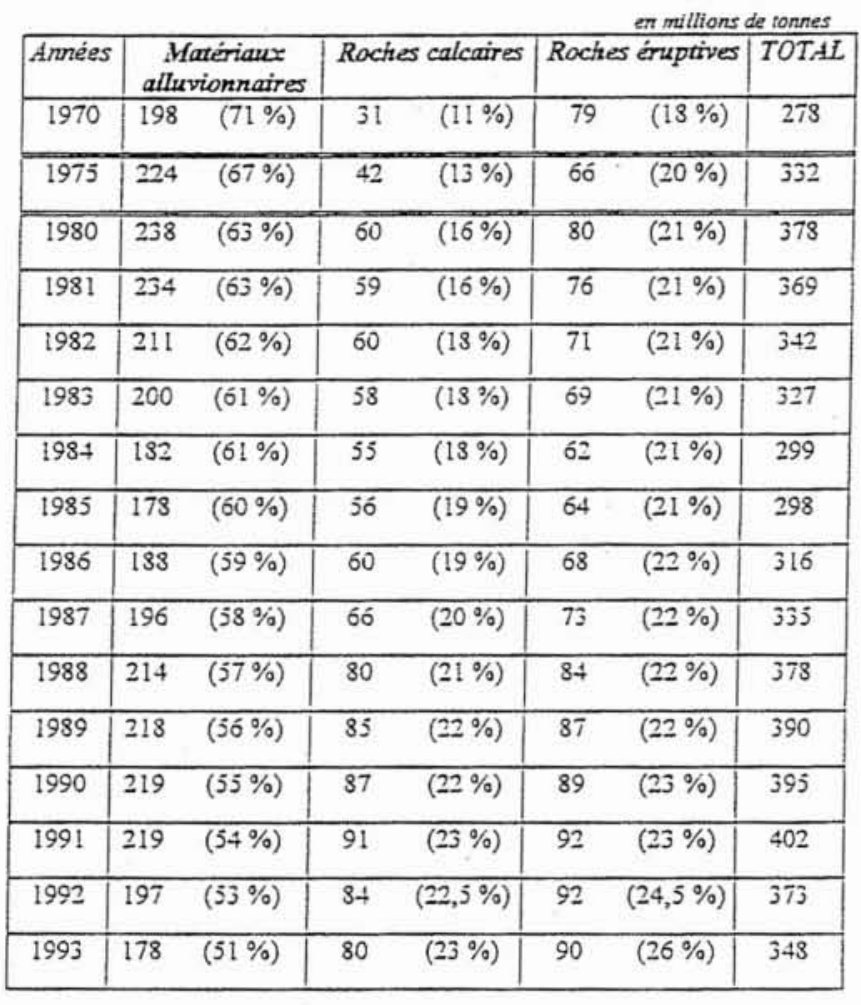

Evolution des extractions de matériaux alluvionnaires en France.

Les matériaux alluvionnaires constituent une ressource fossile, même dans le lit mineur des cours d'eau, car les apports en provenance des hauts bassins sont faibles et bloqués par les barrages construits pour la production d'énergie électrique ou l'écrêtement des crues.

Ils sont actuellement la ressource la plus exploitée pour la production de granulats en France. Les principales raisons en sont : l'importance de cette ressource, sa large répartition sur le territoire, sa grande facilité d'exploitation et le faible coût des extractions, ce qui permet la production de granulats à bas prix.

L'évolution des extractions de matériaux alluvionnaires et la production à partir de roches calcaires et éruptives depuis 1970 est indiquée dans le tableau ci-contre.

rigoureuse. Ces matériaux constituant une ressource fossile, leur usage devrait être réservé à des usages nobles (bétons, revêtement de chaussée) et proscrit pour des remblais. Des recommandations devraient être adressées dans ce sens aux services de l'Etat, ainsi qu'aux établissements publics et aux collectivités locales, qui sont des donneurs d'ordre importants.

- Enfin, la recherche, la production et l'utilisation de matériaux de substitution devraient être encouragées.

\subsection{Les décisions prises à la suite de ce rapport}

Le ministre de l'Environnement a fait part, par lettre du 20 octobre 1995 adressée au préfet coordonnateur du bassin Loire-Bretagne, que les recommandations de ce rapport

recevaient son plein accord. Ce rapport a été diffusé aux services de l'Etat concernés dans le bassin de la Loire, ainsi qu'aux membres du Comité de bassin Loire-Bretagne.

Le projet de SDAGE Loire-Bretagne en tient largement compte.

Ainsi, dans le cadre du Plan-Loire et du SDAGE LoireBretagne, une nouvelle politique se met en place pour la restauration du lit de la Loire, le renforcement de la protection de l'environnement, une exploitation mâtrisée des ressources de matériaux alluvionnaires du lit majeur et la reconstitution des milieux naturels.

Sa mise en oeuvre devrait conduire à une gestion globale du lit de la Loire, à la fois plus naturelle et plus équilibrée, plus écologique et plus respectueuse de l'environnement. 